Proyecciones Journal of Mathematics

Vol. 40, No 5, pp. 1097-1116, October 2021.

Universidad Católica del Norte

Antofagasta - Chile

\title{
k-super cube root cube mean labeling of graphs
}

\author{
V. Princy Kala \\ Holy Cross College (Autonomous), India \\ Received : June 2020. Accepted : January 2021
}

\begin{abstract}
Consider a graph $G$ with $|V(G)|=p$ and $|E(G)|=q$ and let $f: V(G) \rightarrow\{\mathrm{k}, \mathrm{k}+1, \mathrm{k}+2, \ldots \mathrm{p}+\mathrm{q}+\mathrm{k}-1\}\}$ be an injective function. The induced edge labeling $f^{*}$ for a vertex labeling $f$ is defined by $f^{*}(e)=\left\lfloor\sqrt[3]{\frac{f(u)^{3}+f(v)^{3}}{2}}\right\rfloor$ or $\left[\sqrt[3]{\frac{f(u)^{3}+f(v)^{3}}{2}}\right\rfloor$ for all $e=u v \in E(G)$ is bijective. If $f(V(G)) \cup\left\{f^{*}(e): e \in E(G)\right\}=\{\mathrm{k}, \mathrm{k}+1, \mathrm{k}+2, \ldots$, $p+q+k-1\}$, then $f$ is called a $k$-super cube root cube mean labeling. If such labeling exists, then $G$ is a $k$-super cube root cube mean graph. In this paper, I introduce $k$-super cube root cube mean labeling and prove the existence of this labeling to the graphs viz., triangular snake graph $T_{n}$, double triangular snake graph $D\left(T_{n}\right)$, Quadrilateral snake graph $Q_{n}$, double quadrilateral snake graph $D\left(Q_{n}\right)$, alternate triangular snake graph $A\left(T_{n}\right)$, alternate double triangular snake graph $A D\left(T_{n}\right)$, alternate quadrilateral snake graph $A\left(Q_{n}\right)$, $\&$ alternate double quadrilateral snake graph $A D\left(Q_{n}\right)$.
\end{abstract}

Keywords: $k$-super cube root cube mean labeling, $k$-super cube root cube mean graph, snake graph, alternate snake graph.

MSC(2020): 05C78. 


\section{Introduction}

In this paper, all graphs are simple, finite and undirected with $|V(G)|=p$ and $\mid E(G)=q$. Labeling of a graph is an assignment of integers to the vertices or edges or both subject to certain conditions. For detailed study of graph labeling, refer to J. A. Gallian [2]. Many researchers in practice contributed various types of labeling like k-super mean labeling [3,7], root square mean labeling [6], k-super root square mean labeling [1], cube root cube mean labeling [4], super cube root cube mean labeling [5], etc. In this paper, I have added another type of graph labeling, i.e., k-super cube root cube mean labeling. Consider a graph $G$ with $|V(G)|=p$ and $|E(G)|=q$ and let $f: V(G) \rightarrow\{\mathrm{k}, \mathrm{k}+1, \mathrm{k}+2, \ldots, \mathrm{p}+\mathrm{q}+\mathrm{k}-1\}$ be an injective function. The induced edge labeling $f^{*}$ for a vertex labeling $f$ is defined by $f^{*}(\mathrm{e})=\left\lfloor\sqrt[3]{\frac{f(u)^{3}+f(v)^{3}}{2}}\right\rfloor$ or $\left\lceil\frac{\sqrt[3]{\frac{f(u)^{3}+f(v)^{3}}{2}}}{2}\right.$ for all $e=u v \in E(G)$ is bijective. If $f(V(G)) \cup\left\{f^{*}(e): e \in E(G)\right\}=$ $\{\mathrm{k}, \mathrm{k}+1, \mathrm{k}+2, \ldots, \mathrm{p}+\mathrm{q}+\mathrm{k}-1\}$, then $f$ is known as $\mathrm{k}$-super cube root cube mean labeling. If such labeling exists, then $G$ is a k-super cube root cube mean graph. In this paper, it is assumed that $k$ is an integer and its value is $\geq 1$.

\section{Preliminaries}

Definition 2.1. The triangular snake graph $T_{n}$ is obtained from a path $P_{n}$ by replacing each edge of the path by a triangle $C_{3}$. That is a triangular snake graph is obtained from a path $u_{1}, u_{2}, \ldots, u_{n}$ by joining $u_{i}$ and $u_{i+1}$ to a new vertex $v_{i}, 1 \leq i \leq n-1$.

Definition 2.2. An alternate triangular snake graph $A\left(T_{n}\right)$ is obtained from a path $u_{1}, u_{2}, \ldots, u_{n}$ by joining $u_{i}$ and $u_{i+1}$ (alternately) to a new vertex $v_{i}$. That is every alternate edge of a path is replaced by a triangle $C_{3}$.

Definition 2.3. A double triangular snake graph $D\left(T_{n}\right)$ consists of two triangular snakes that have a common path.

Definition 2.4. An alternate double triangular snake graph $A D\left(T_{n}\right)$ consists of two alternate triangular snakes having a common path. That is to construct an alternate double triangular snake graph $A D\left(T_{n}\right)$, we have to 
join $u_{i}$ and $u_{i+1}, 1 \leq i \leq n-1$ (alternately) from a path with vertices $u_{1}$, $u_{2}, \ldots, u_{n}$ to the vertices $v_{j}$ and $w_{j}, 1 \leq j \leq\left\lfloor\frac{n}{2}\right\rfloor$.

Definition 2.5. A quadrilateral snake graph $Q_{n}$ is obtained from a path $P_{n}$ by replacing each edge of the path by a cycle $C_{4}$. That is a quadrilateral snake graph $Q_{n}$ is obtained from a path $u_{1}, u_{2}, \ldots, u_{n}$ by joining $u_{i}$, $u_{i+1}$ to new vertices $v_{i}$ and $w_{i}$ respectively and adding edges $v_{i} w_{i}$ for $i=$ $1,2, \ldots ., n-1$.

Definition 2.6. To construct an alternate quadrilateral snake graph $A\left(Q_{n}\right)$, we have to join $u_{i}$ and $u_{i+1}$ alternately from a path with vertices $u_{1}, u_{2}$, $\ldots, u_{n}$ to the vertices $v_{j}, w_{j}$ respectively then joining $v_{j}$ and $w_{j}, 1 \leq i$ $\leq n-1 \& 1 \leq j \leq\left\lfloor\frac{n}{2}\right\rfloor$. That is every alternate edge of a path is replaced by a cycle $C_{4}$.

Definition 2.7. A double quadrilateral snake graph $D\left(Q_{n}\right)$ is obtained from two quadrilateral snakes that have a common path.

Definition 2.8. An alternate double quadrilateral snake graph $A D\left(Q_{n}\right)$ is obtained from two alternative quadrilateral snakes that have a common path.

\section{Main Results}

Theorem 3.1. Any triangular snake graph $T_{n}$ is a $k$-super cube root cube mean graph.

Proof. Let $\mathrm{T}_{n}$ be a triangular snake graph.

Here $\mathrm{p}=2 \mathrm{n}-1 \& \mathrm{q}=3 \mathrm{n}-3$

Hence $\mathrm{p}+\mathrm{q}=5 \mathrm{n}-4$.

Define a function $\mathrm{f}: \mathrm{V}\left(\mathrm{T}_{n}\right) \rightarrow\{\mathrm{k}, \mathrm{k}+1, \mathrm{k}+2, \ldots, \mathrm{p}+\mathrm{q}+\mathrm{k}-1\}$ by

$\mathrm{f}\left(\mathrm{u}_{i}\right)=\mathrm{k}+5 \mathrm{i}-5, \quad 1 \leq \mathrm{i} \leq \mathrm{n}$

$\mathrm{f}(\mathrm{vi})=\mathrm{k}+5 \mathrm{i}-3,1 \leq \mathrm{i} \leq \mathrm{n}-1$.

Then, the edge labels of $\mathrm{T}_{n}$ are

$\mathrm{f}^{*}\left(\mathrm{u}_{i} \mathrm{v}_{i}\right)=\mathrm{k}+5 \mathrm{i}-4,1 \leq \mathrm{i} \leq \mathrm{n}-1$

$\mathrm{f}^{*}\left(\mathrm{u}_{i} \mathrm{u}_{i+1}\right)=\mathrm{k}+5 \mathrm{i}-2,1 \leq \mathrm{i} \leq \mathrm{n}-1$

$\mathrm{f}^{*}\left(\mathrm{u}_{i+1} \mathrm{v}_{i}\right)=\mathrm{k}+5 \mathrm{i}-1,1 \leq \mathrm{i} \leq \mathrm{n}-1$.

Hence $\mathrm{f}\left(\mathrm{V}\left(\mathrm{T}_{n}\right)\right) \cup\left\{\mathrm{f}^{*}(\mathrm{e}): \mathrm{e} \in \mathrm{E}\left(\mathrm{T}_{n}\right)\right\}=\{\mathrm{k}, \mathrm{k}+1, \mathrm{k}+2, \ldots, \mathrm{p}+\mathrm{q}+\mathrm{k}-1\}$.

Therefore any Triangular snake graph $\mathrm{T}_{n}$ is a k-super cube root cube mean graph. 
An example of 400-super cube root cube mean labeling of $T_{5}$ is shown in Figure 1

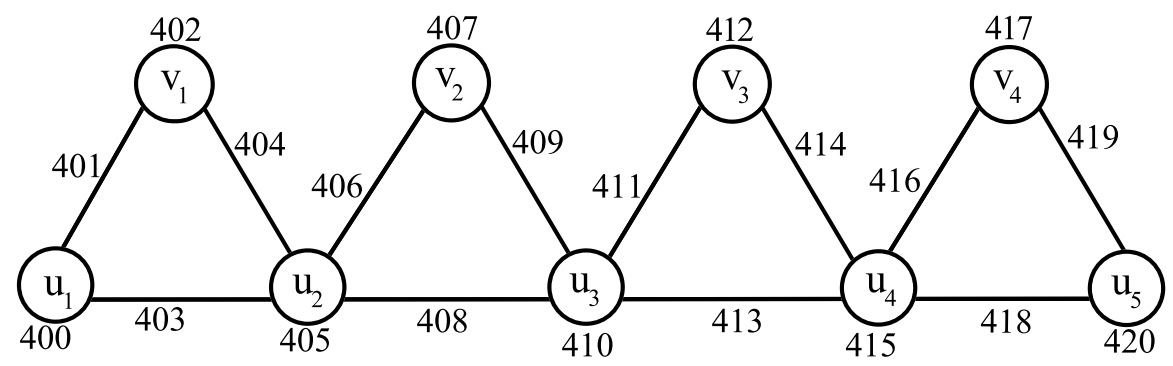

Figure 1: 400-super cube root cube mean labeling of $T_{5}$

Theorem 3.2. Any alternate triangular snake graph $A\left(T_{n}\right)$ is a $k$-super cube root cube mean graph.

Proof. Let $\mathrm{A}\left(\mathrm{T}_{n}\right)$ be an alternate triangular snake graph.

In this theorem, consider two cases.

Case 1. The triangle in $\mathrm{A}\left(\mathrm{T}_{n}\right)$ starts from $\mathrm{u}_{1}$

In this case

$\mathrm{p}=\left\{\begin{array}{l}\frac{3 n}{2}, \text { if } n \text { is even } \\ \frac{3 n-1}{2}, \text { if } n \text { is odd. }\end{array}\right.$

$\& \mathrm{q}=\left\{\begin{array}{l}2 n-1, \text { if } n \text { is even; } \\ 2 n-2, \text { if } n \text { is odd }\end{array}\right.$

Hence $\mathrm{p}+\mathrm{q}= \begin{cases}\frac{7 n-2}{2}, & \text { if } n \text { is even; } \\ \frac{7 n-5}{2}, & \text { if } n \text { is odd. }\end{cases}$

Define a function $\mathrm{f}: \mathrm{V}\left(\mathrm{A}\left(\mathrm{T}_{n}\right)\right) \rightarrow\{\mathrm{k}, \mathrm{k}+1, \mathrm{k}+2, \ldots, \mathrm{p}+\mathrm{q}+\mathrm{k}-1\}$ by

$\mathrm{f}\left(\mathrm{u}_{2 i-1}\right)=\mathrm{k}+7 \mathrm{i}-7,1 \leq \mathrm{i} \leq \frac{n}{2}$ if $\mathrm{n}$ is even $\& 1 \leq \mathrm{i} \leq \frac{n+1}{2}$ if $\mathrm{n}$ is odd. $\mathrm{f}\left(\mathrm{u}_{2 i}\right)=\mathrm{k}+7 \mathrm{i}-2,1 \leq \mathrm{i} \leq \frac{n}{2}$ if $\mathrm{n}$ is even $\& 1 \leq \mathrm{i} \leq \frac{n-1}{2}$ if $\mathrm{n}$ is odd. 
$\mathrm{f}\left(\mathrm{v}_{i}\right)=\mathrm{k}+7 \mathrm{i}-5,1 \leq \mathrm{i} \leq \frac{n}{2}$ if $\mathrm{n}$ is even $\& 1 \leq \mathrm{i} \leq \frac{n-1}{2}$ if $\mathrm{n}$ is odd.

Then, the edge labels of $\mathrm{A}\left(\mathrm{T}_{n}\right)$ are

$\mathrm{f}^{*}\left(\mathrm{u}_{2 i-1} \mathrm{u}_{2 i}\right)=\mathrm{k}+7 \mathrm{i}-4,1 \leq \mathrm{i} \leq \frac{n}{2}$ if $\mathrm{n}$ is even $\& 1 \leq \mathrm{i} \leq \frac{n-1}{2}$ if $\mathrm{n}$ is odd. $\mathrm{f}^{*}\left(\mathrm{u}_{2 i} \mathrm{u}_{2 i+1}\right)=\mathrm{k}+7 \mathrm{i}-1,1 \leq \mathrm{i} \leq \frac{n-2}{2}$ if $\mathrm{n}$ is even $\& 1 \leq \mathrm{i} \leq \frac{n-1}{2}$ if $\mathrm{n}$ is odd. $\mathrm{f}^{*}\left(\mathrm{u}_{2 i-1} \mathrm{v}_{i}\right)=\mathrm{k}+7 \mathrm{i}-6,1 \leq \mathrm{i} \leq \frac{n}{2}$ if $\mathrm{n}$ is even $\& 1 \leq \mathrm{i} \leq \frac{n-1}{2}$ if $\mathrm{n}$ is odd. $\mathrm{f}^{*}\left(\mathrm{u}_{2 i} \mathrm{v}_{i}\right)=\mathrm{k}+7 \mathrm{i}-3, \quad 1 \leq \mathrm{i} \leq \frac{n}{2}$ if $\mathrm{n}$ is even $\& 1 \leq \mathrm{i} \leq \frac{n-1}{2}$ if $\mathrm{n}$ is odd. Hence $\mathrm{f}\left[\mathrm{V}\left(\mathrm{A}\left(\mathrm{T}_{n}\right)\right)\right] \cup\left\{\mathrm{f}^{*}(\mathrm{e}): \mathrm{e} \in \mathrm{E}\left(\mathrm{A}\left(\mathrm{T}_{n}\right)\right)\right\}=\{\mathrm{k}, \mathrm{k}+1, \mathrm{k}+2, \ldots, \mathrm{p}+\mathrm{q}+\mathrm{k}-1\}$. An example of 15 -super cube root cube mean labeling of $\mathrm{A}\left(\mathrm{T}_{8}\right)$ [Triangle start from $\left.\mathrm{u}_{1}\right]$ is shown in Figure 2.

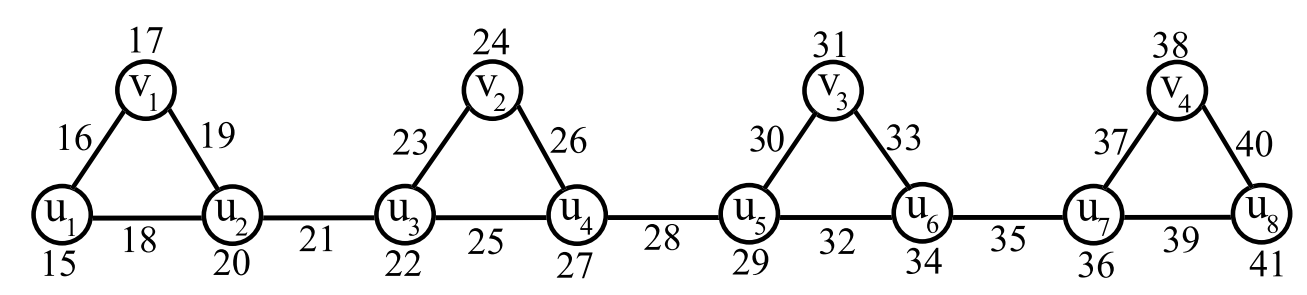

Figure 2: 15-super cube root cube mean labeling of $A\left(T_{8}\right)$ [Triangle start from $u_{1}$ ]

Case 2. The triangle in $A\left(T_{n}\right)$ starts from $\mathrm{u}_{2}$

In this case $\mathrm{p}= \begin{cases}\frac{3 n-2}{2}, & \text { if } n \text { is even; } \\ \frac{3 n-1}{2}, & \text { if } n \text { is odd. }\end{cases}$

$\& \mathrm{q}=\left\{\begin{array}{l}2 n-3, \text { if } n \text { is even } \\ 2 n-2, \text { if } n \text { is odd }\end{array}\right.$

Hence $\mathrm{p}+\mathrm{q}= \begin{cases}\frac{7 n-8}{2}, & \text { if } n \text { is even } \\ \frac{7 n-5}{2}, & \text { if } n \text { is odd }\end{cases}$

Define a function $\mathrm{f}: \mathrm{V}\left(\mathrm{A}\left(\mathrm{T}_{n}\right)\right) \rightarrow\{\mathrm{k}, \mathrm{k}+1, \mathrm{k}+2, \ldots, \mathrm{p}+\mathrm{q}+\mathrm{k}-1\}$ by

$\mathrm{f}\left(\mathrm{u}_{2 i-1}\right)=\mathrm{k}+7 \mathrm{i}-7, \quad 1 \leq \mathrm{i} \leq \frac{n}{2}$ if $\mathrm{n}$ is even $\& 1 \leq \mathrm{i} \leq \frac{n+1}{2}$ if $\mathrm{n}$ is odd. $\mathrm{f}\left(\mathrm{u}_{2 i}\right)=\mathrm{k}+7 \mathrm{i}-5,1 \leq \mathrm{i} \leq \frac{n}{2}$ if $\mathrm{n}$ is even $\& 1 \leq \mathrm{i} \leq \frac{n-1}{2}$ if $\mathrm{n}$ is odd. 
$\mathrm{f}\left(\mathrm{v}_{i}\right)=\mathrm{k}+7 \mathrm{i}-3,1 \leq \mathrm{i} \leq \frac{n-2}{2}$ if $\mathrm{n}$ is even $\& 1 \leq \mathrm{i} \leq \frac{n-1}{2}$ if $\mathrm{n}$ is odd.

Then, the edge labels of $\mathrm{A}\left(\mathrm{T}_{n}\right)$ are

$\mathrm{f}^{*}\left(\mathrm{u}_{2 i-1} \mathrm{u}_{2 i}\right)=\mathrm{k}+7 \mathrm{i}-6,1 \leq \mathrm{i} \leq \frac{n}{2}$ if $\mathrm{n}$ is even $\& 1 \leq \mathrm{i} \leq \frac{n-1}{2}$ if $\mathrm{n}$ is odd. $\mathrm{f}^{*}\left(\mathrm{u}_{2 i} \mathrm{u}_{2 i+1}\right)=\mathrm{k}+7 \mathrm{i}-2, \quad 1 \leq \mathrm{i} \leq \frac{n-2}{2}$ if $\mathrm{n}$ is even $\& 1 \leq \mathrm{i} \leq \frac{n-1}{2}$ if $\mathrm{n}$ is odd.

$\mathrm{f}^{*}\left(\mathrm{u}_{2 i} \mathrm{v}_{i}\right)=\mathrm{k}+7 \mathrm{i}-4,1 \leq \mathrm{i} \leq \frac{n-2}{2}$ if $\mathrm{n}$ is even $\& 1 \leq \mathrm{i} \leq \frac{n-1}{2}$ if $\mathrm{n}$ is odd. $\mathrm{f}^{*}\left(\mathrm{u}_{2 i+1} \mathrm{v}_{i}\right)=\mathrm{k}+7 \mathrm{i}-1,1 \leq \mathrm{i} \leq \frac{n-2}{2}$ if $\mathrm{n}$ is even $\& 1 \leq \mathrm{i} \leq \frac{n-1}{2}$ if $\mathrm{n}$ is odd. Hence $\mathrm{f}\left[\mathrm{V}\left(\mathrm{A}\left(\mathrm{T}_{n}\right)\right)\right] \cup\left\{\mathrm{f}^{*}(\mathrm{e}): \mathrm{e} \in \mathrm{E}\left(\mathrm{A}\left(\mathrm{T}_{n}\right)\right)\right\}=\{\mathrm{k}, \mathrm{k}+1, \mathrm{k}+2, \ldots, \mathrm{p}+\mathrm{q}+\mathrm{k}-1\}$. An example of 15 -super cube root cube mean labeling of $A\left(T_{7}\right)$ [Triangle start from $u_{2}$ ] is shown in Figure 3.

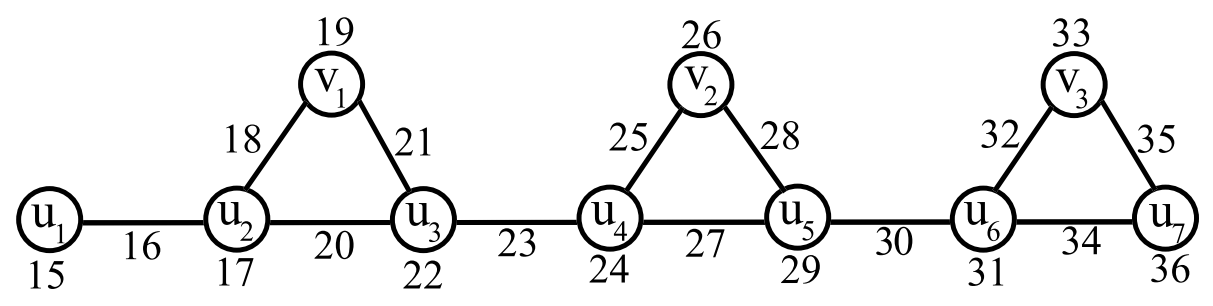

Figure 3: 15-super cube root cube mean labeling of $A\left(T_{7}\right)$ [Triangle start from $u_{2}$ ]

From the above cases, an alternate triangular snake graph $A\left(T_{n}\right)$ is a k-super cube root cube mean graph.

Theorem 3.3. Any double triangular snake graph $D\left(T_{n}\right)$ is a $k$-super cube root cube mean graph.

Proof. Let $\mathrm{D}\left(\mathrm{T}_{n}\right)$ be a double triangular snake graph.

Here $\mathrm{p}=3 \mathrm{n}-2 \& \mathrm{q}=5 \mathrm{n}-5$ Hence $\mathrm{p}+\mathrm{q}=8 \mathrm{n}-7$.

Define a function $\mathrm{f}: \mathrm{V}\left(\mathrm{D}\left(\mathrm{T}_{n}\right)\right) \rightarrow\{\mathrm{k}, \mathrm{k}+1, \mathrm{k}+2, \ldots, \mathrm{p}+\mathrm{q}+\mathrm{k}-1\}$ by $\mathrm{f}\left(\mathrm{u}_{1}\right)=\mathrm{k}$, for all $\mathrm{k}$.

$\mathrm{f}\left(\mathrm{u}_{2}\right)=\left\{\begin{array}{l}k+5, k=1,2,3, \ldots 10 \\ k+6, \text { otherwise. }\end{array}\right.$ 
$\mathrm{f}\left(\mathrm{u}_{i}\right)=\mathrm{k}+8 \mathrm{i}-10,3 \leq \mathrm{i} \leq \mathrm{n}$

$\mathrm{f}\left(\mathrm{v}_{i}\right)=\mathrm{k}+8 \mathrm{i}, \quad 1 \leq \mathrm{i} \leq \mathrm{n}-1$

$\mathrm{f}\left(\mathrm{w}_{i}\right)=\mathrm{k}+8 \mathrm{i}-6,1 \leq \mathrm{i} \leq \mathrm{n}-1$.

Then, the edge labels of $\mathrm{D}\left(\mathrm{T}_{n}\right)$ are

$\mathrm{f}^{*}\left(\mathrm{u}_{i} \mathrm{u}_{i+1}\right)=\mathrm{k}+8 \mathrm{i}-5,1 \leq \mathrm{i} \leq \mathrm{n}-1$.

$\mathrm{f}^{*}\left(\mathrm{u}_{1} \mathrm{v}_{1}\right)=\left\{\begin{array}{l}k+6, k=1,2,3, \ldots 10 \\ k+4, \text { otherwise }\end{array}\right.$

$\mathrm{f}^{*}\left(\mathrm{u}_{i} \mathrm{v}_{i}\right)=\mathrm{k}+8 \mathrm{i}-4,2 \leq \mathrm{i} \leq \mathrm{n}-1$

$\mathrm{f}^{*}\left(\mathrm{u}_{i+1} \mathrm{v}_{i}\right)=\mathrm{k}+8 \mathrm{i}-1,1 \leq \mathrm{i} \leq \mathrm{n}-1$

$\mathrm{f}^{*}\left(\mathrm{u}_{i} \mathrm{w}_{i}\right)=\mathrm{k}+8 \mathrm{i}-7,1 \leq \mathrm{i} \leq \mathrm{n}-1$

$\mathrm{f}^{*}\left(\mathrm{u}_{2} \mathrm{w}_{1}\right)=\left\{\begin{array}{l}k+4, k=1,2,3, \ldots 10 \\ k+5, \text { otherwise. }\end{array}\right.$

$\mathrm{f}^{*}\left(\mathrm{u}_{i+1} \mathrm{w}_{i}\right)=\mathrm{k}+8 \mathrm{i}-3,2 \leq \mathrm{i} \leq \mathrm{n}-1$.

Hence $\mathrm{f}\left[\mathrm{V}\left(\mathrm{D}\left(\mathrm{T}_{n}\right)\right)\right] \cup\left\{\mathrm{f}^{*}(\mathrm{e}): \mathrm{e} \in \mathrm{E}\left(\mathrm{D}\left(\mathrm{T}_{n}\right)\right)\right\}=\{\mathrm{k}, \mathrm{k}+1, \mathrm{k}+2, \ldots, \mathrm{p}+\mathrm{q}+\mathrm{k}-1\}$.

Therefore any double triangular snake graph $\mathrm{D}\left(\mathrm{T}_{n}\right)$ is a k-super cube root

cube mean graph.

An example of 75-super cube root cube mean labeling of $D\left(T_{5}\right)$ is shown

in Figure 4.

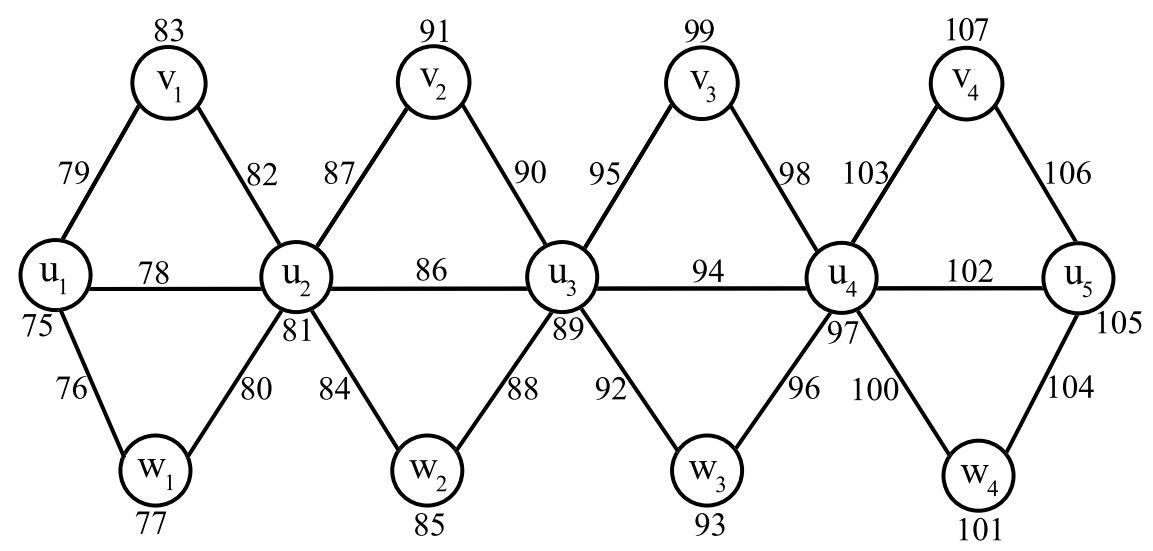

Figure 4: 75-super cube root cube mean labeling of $D\left(T_{5}\right)$

Theorem 3.4. Any alternate double triangular snake graph $A D\left(T_{n}\right)$ is a $k$-super cube root cube mean graph. 
Proof. Let $\mathrm{AD}(\mathrm{T} n)$ be an alternate double triangular snake graph. In this theorem, consider two cases.

Case 1. The triangle in $\operatorname{AD}\left(\mathrm{T}_{n}\right)$ starts from $\mathrm{u}_{1}$

In this case $\mathrm{p}=\left\{\begin{array}{l}2 n, \text { if } n \text { is even; } \\ 2 n-1, \text { if } n \text { is odd. }\end{array}\right.$

$\& \mathrm{q}=\left\{\begin{array}{l}3 n-1, \text { if } n \text { is even } \\ 3 n-3, \text { if } n \text { is odd. }\end{array}\right.$

Hence $\mathrm{p}+\mathrm{q}=\left\{\begin{array}{l}5 n-1, \text { if } n \text { is even; } \\ 5 n-4, \text { if } n \text { is odd. }\end{array}\right.$

Define a function $\mathrm{f}: \mathrm{V}\left(\mathrm{AD}\left(\mathrm{T}_{n}\right)\right) \rightarrow\{\mathrm{k}, \mathrm{k}+1, \mathrm{k}+2, \ldots, \mathrm{p}+\mathrm{q}+\mathrm{k}-1\}$ by $\mathrm{f}\left(\mathrm{u}_{2 i-1}\right)=\mathrm{k}+10 \mathrm{i}-8,1 \leq \mathrm{i} \leq \frac{n}{2}$ if $\mathrm{n}$ is even $\& 1 \leq \mathrm{i} \leq \frac{n+1}{2}$ if $\mathrm{n}$ is odd. $\mathrm{f}\left(\mathrm{u}_{2 i}\right)=\mathrm{k}+10 \mathrm{i}-5,1 \leq \mathrm{i} \leq \frac{n}{2}$ if $\mathrm{n}$ is even $\& 1 \leq \mathrm{i} \leq \frac{n-1}{2}$ if $\mathrm{n}$ is odd. $\mathrm{f}\left(\mathrm{v}_{i}\right)=\mathrm{k}+10 \mathrm{i}-2, \quad 1 \leq \mathrm{i} \leq \frac{n}{2}$ if $\mathrm{n}$ is even $\& 1 \leq \mathrm{i} \leq \frac{n-1}{2}$ if $\mathrm{n}$ is odd. $\mathrm{f}\left(\mathrm{w}_{i}\right)=\mathrm{k}+10 \mathrm{i}-10,1 \leq \mathrm{i} \leq \frac{n}{2}$ if $\mathrm{n}$ is even $\& 1 \leq \mathrm{i} \leq \frac{n-1}{2}$ if $\mathrm{n}$ is odd. Then, the edge labels of $\operatorname{AD}\left(\mathrm{T}_{n}\right)$ are $\mathrm{f}^{*}\left(\mathrm{u}_{2 i-1} \mathrm{u}_{2 i}\right)=\mathrm{k}+10 \mathrm{i}-6,1 \leq \mathrm{i} \leq \frac{n}{2}$ if $\mathrm{n}$ is even $\& 1 \leq \mathrm{i} \leq \frac{n-1}{2}$ if $\mathrm{n}$ is odd. $\mathrm{f}^{*}\left(\mathrm{u}_{2 i} \mathrm{u}_{2 i+1}\right)=\mathrm{k}+10 \mathrm{i}-1,1 \leq \mathrm{i} \leq \frac{n-2}{2}$ if $\mathrm{n}$ is even $\& 1 \leq \mathrm{i} \leq \frac{n-1}{2}$ if $\mathrm{n}$ is odd.

$\mathrm{f}^{*}\left(\mathrm{u}_{2 i-1} \mathrm{v}_{i}\right)=\mathrm{k}+10 \mathrm{i}-4,1 \leq \mathrm{i} \leq \frac{n}{2}$ if $\mathrm{n}$ is even $\& 1 \leq \mathrm{i} \leq \frac{n-1}{2}$ if $\mathrm{n}$ is odd. $\mathrm{f}^{*}\left(\mathrm{u}_{2 i} \mathrm{v}_{i}\right)=\mathrm{k}+10 \mathrm{i}-3,1 \leq \mathrm{i} \leq \frac{n}{2}$ if $\mathrm{n}$ is even $\& 1 \leq \mathrm{i} \leq \frac{n-1}{2}$ if $\mathrm{n}$ is odd. $\mathrm{f}^{*}\left(\mathrm{u}_{2 i-1} \mathrm{w}_{i}\right)=\mathrm{k}+10 \mathrm{i}-9,1 \leq \mathrm{i} \leq \frac{n}{2}$ if $\mathrm{n}$ is even $\& 1 \leq \mathrm{i} \leq \frac{n-1}{2}$ if $\mathrm{n}$ is odd. $\mathrm{f}^{*}\left(\mathrm{u}_{2 i} \mathrm{w}_{i}\right)=\mathrm{k}+10 \mathrm{i}-7,1 \leq \mathrm{i} \leq \frac{n}{2}$ if $\mathrm{n}$ is even $\& 1 \leq \mathrm{i} \leq \frac{n-1}{2}$ if $\mathrm{n}$ is odd.

Hence $\mathrm{f}\left[\mathrm{V}\left(\mathrm{AD}\left(\mathrm{T}_{n}\right)\right)\right] \cup\left\{\mathrm{f}^{*}(\mathrm{e}): \mathrm{e} \in \mathrm{E}\left(\mathrm{AD}\left(\mathrm{T}_{n}\right)\right)\right\}=\{k, k+1, k+2, \ldots, p+q+k-1\}$. An example of 50-super cube root cube mean labeling of $\operatorname{AD}\left(\mathrm{T}_{8}\right)$ [triangle

start from $\mathrm{u}_{1}$ ] is shown in Figure 5 . 


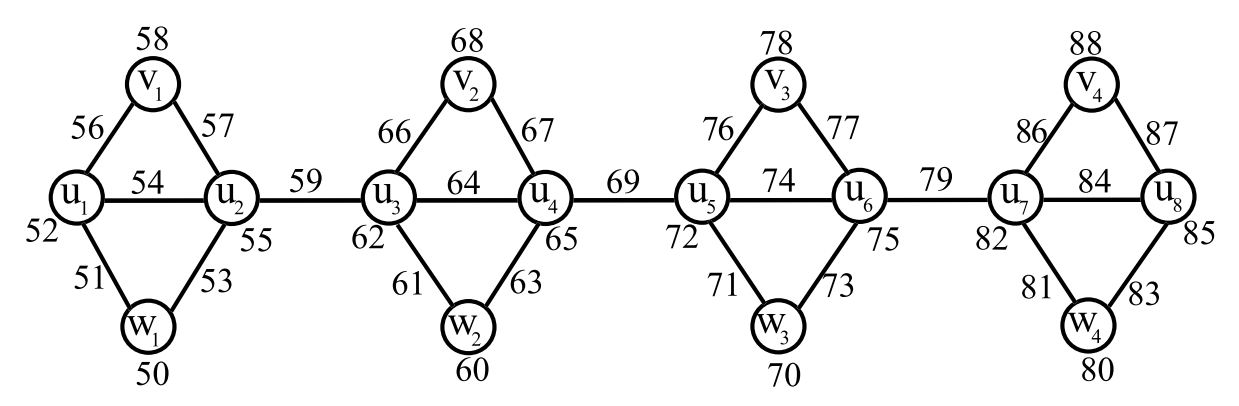

Figure 5: 50-super cube root cube mean labeling of $A D\left(T_{8}\right)$ [triangle start from $u_{1}$ ]

Case 2. The triangle in $\operatorname{AD}\left(\mathrm{T}_{n}\right)$ starts from $\mathrm{u}_{2}$

In this case $\mathrm{p}=\left\{\begin{array}{l}2 n-2, \text { if } n \text { is even; } \\ 2 n-1, \text { if } n \text { is odd. }\end{array}\right.$

$\& \mathrm{q}=\left\{\begin{array}{l}3 n-5, \text { if } n \text { is even } \\ 3 n-3, \text { if } n \text { is odd }\end{array}\right.$

Hence $\mathrm{p}+\mathrm{q}=\left\{\begin{array}{l}5 n-7, \text { if } n \text { is even; } \\ 5 n-4, \text { if } n \text { is odd. }\end{array}\right.$

Define a function $\mathrm{f}: \mathrm{V}\left(\mathrm{AD}\left(\mathrm{T}_{n}\right)\right) \rightarrow\{\mathrm{k}, \mathrm{k}+1, \mathrm{k}+2, \ldots, \mathrm{p}+\mathrm{q}+\mathrm{k}-1\}$

by

$\mathrm{f}\left(\mathrm{u}_{2 i-1}\right)=\mathrm{k}+10 \mathrm{i}-10,1 \leq \mathrm{i} \leq \frac{n}{2}$ if $\mathrm{n}$ is even $\& 1 \leq \mathrm{i} \leq \frac{n+1}{2}$ if $\mathrm{n}$ is odd. $\mathrm{f}\left(\mathrm{u}_{2 i}\right)=\mathrm{k}+10 \mathrm{i}-8,1 \leq \mathrm{i} \leq \frac{n}{2}$ if $\mathrm{n}$ is even $\& 1 \leq \mathrm{i} \leq \frac{n-1}{2}$ if $\mathrm{n}$ is odd.

$\mathrm{f}\left(\mathrm{v}_{i}\right)=\mathrm{k}+10 \mathrm{i}-4,1 \leq \mathrm{i} \leq \frac{n-2}{2}$ if $\mathrm{n}$ is even $\& 1 \leq \mathrm{i} \leq \frac{n-1}{2}$ if $\mathrm{n}$ is odd. $\mathrm{f}\left(\mathrm{w}_{i}\right)=\mathrm{k}+10 \mathrm{i}-5,1 \leq \mathrm{i} \leq \frac{n-2}{2}$ if $\mathrm{n}$ is even $\& 1 \leq \mathrm{i} \leq \frac{n-1}{2}$ if $\mathrm{n}$ is odd.

Then, the edge labels of $\mathrm{AD}\left(\mathrm{T}_{n}\right)$ are

$\mathrm{f}^{*}\left(\mathrm{u}_{2 i-1} \mathrm{u}_{2 i}\right)=\mathrm{k}+10 \mathrm{i}-9,1 \leq \mathrm{i} \leq \frac{n}{2}$ if $\mathrm{n}$ is even $\& 1 \leq \mathrm{i} \leq \frac{n-1}{2}$ if $\mathrm{n}$ is odd. $\mathrm{f}^{*}\left(\mathrm{u}_{2 i} \mathrm{u}_{2 i+1}\right)=\mathrm{k}+10 \mathrm{i}-3,1 \leq \mathrm{i} \leq \frac{n-2}{2}$ if $\mathrm{n}$ is even $\& 1 \leq \mathrm{i} \leq \frac{n-1}{2}$ if $\mathrm{n}$ is odd.

$\mathrm{f}^{*}\left(\mathrm{u}_{2 i} \mathrm{v}_{i}\right)=\mathrm{k}+10 \mathrm{i}-6,1 \leq \mathrm{i} \leq \frac{n-2}{2}$ if $\mathrm{n}$ is even $\& 1 \leq \mathrm{i} \leq \frac{n-1}{2}$ if $\mathrm{n}$ is odd. $\mathrm{f}^{*}\left(\mathrm{u}_{2 i+1} \mathrm{v}_{i}\right)=\mathrm{k}+10 \mathrm{i}-1,1 \leq \mathrm{i} \leq \frac{n-2}{2}$ if $\mathrm{n}$ is even\& $1 \leq \mathrm{i} \leq \frac{n-1}{2}$ if $\mathrm{n}$ is odd. $\mathrm{f}^{*}\left(\mathrm{u}_{2 i+1} \mathrm{w}_{i}\right)=\mathrm{k}+10 \mathrm{i}-2,1 \leq \overline{\mathrm{i}} \leq \frac{n-2}{2}$ if $\mathrm{n}$ is even $\& 1 \leq \mathrm{i} \leq \frac{n-1}{2}$ if $\mathrm{n}$ is odd. 
$\mathrm{f}^{*}\left(\mathrm{u}_{2 i} \mathrm{w}_{i}\right)=\mathrm{k}+10 \mathrm{i}-7,1 \leq \mathrm{i} \leq \frac{n-2}{2}$ if $\mathrm{n}$ is even $\& 1 \leq \mathrm{i} \leq \frac{n-1}{2}$ if $\mathrm{n}$ is odd.

Hence $\mathrm{f}\left[\mathrm{V}\left(\mathrm{AD}\left(\mathrm{T}_{n}\right)\right)\right] \cup\left\{\mathrm{f}^{*}(\mathrm{e}): \mathrm{e} \in \mathrm{E}\left(\mathrm{AD}\left(\mathrm{T}_{n}\right)\right)\right\}=\{\mathrm{k}, \mathrm{k}+1, \mathrm{k}+2, \ldots, \mathrm{p}+\mathrm{q}+\mathrm{k}-1\}$. An example of 50-super cube root cube mean labeling of $A D\left(T_{7}\right)$ [triangle start from $\left.u_{2}\right]$ is shown in Figure 6.

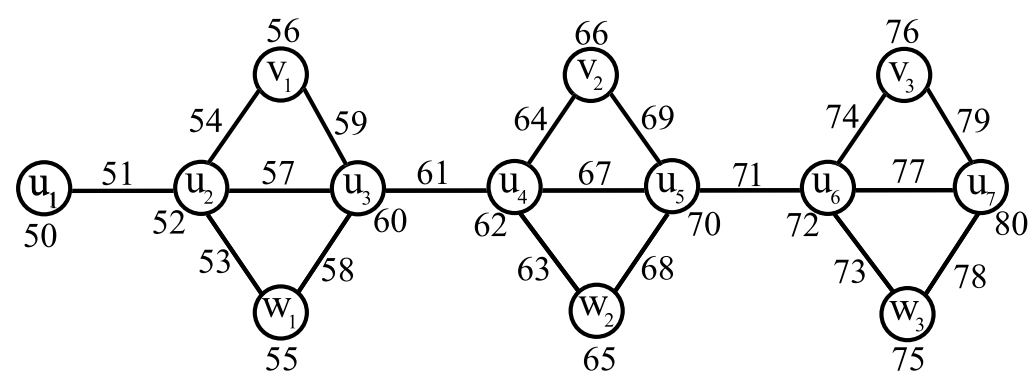

Figure 6: 50-super cube root cube mean labeling of $A D\left(T_{7}\right)$ [triangle start from $u_{2}$ ]

From the above cases, an alternate double triangular snake graph $\mathrm{AD}\left(\mathrm{T}_{n}\right)$ is a k-super cube root cube mean graph

Theorem 3.5. Any Quadrilateral snake graph $Q_{n}$ is a k-super cube root cube mean graph.

Proof. Let $\mathrm{Q}_{n}$ be a quadrilateral snake graph.

Here $\mathrm{p}=3 \mathrm{n}-2 \& \mathrm{q}=4 \mathrm{n}-4$ Hence $\mathrm{p}+\mathrm{q}=7 \mathrm{n}-6$.

Define a function $\mathrm{f}: \mathrm{V}\left(\mathrm{Q}_{n}\right) \rightarrow\{\mathrm{k}, \mathrm{k}+1, \mathrm{k}+2, \ldots, \mathrm{p}+\mathrm{q}+\mathrm{k}-1\}$ by $\mathrm{f}\left(\mathrm{u}_{i}\right)=\mathrm{k}+7 \mathrm{i}-7,1 \leq \mathrm{i} \leq \mathrm{n}$

$\mathrm{f}\left(\mathrm{v}_{i}\right)=\mathrm{k}+7 \mathrm{i}-5,1 \leq \mathrm{i} \leq \mathrm{n}-1$

$\mathrm{f}\left(\mathrm{w}_{1}\right)= \begin{cases}k+4, & k=1,2,3 \\ k+5, & \text { otherwise. }\end{cases}$

$\mathrm{f}\left(\mathrm{w}_{i}\right)=\mathrm{k}+7 \mathrm{i}-2,2 \leq \mathrm{i} \leq \mathrm{n}-1$.

Then, the edge labels of $Q_{n}$ are $\mathrm{f}^{*}\left(\mathrm{u}_{1} \mathrm{u}_{2}\right)=\left\{\begin{array}{l}k+5, k=1,2,3 \\ k+4, \text { otherwise. }\end{array}\right.$ 
$\mathrm{f}^{*}\left(\mathrm{u}_{i} \mathrm{u}_{i+1}\right)=\mathrm{k}+7 \mathrm{i}-3,2 \leq \mathrm{i} \leq \mathrm{n}-1$

$\mathrm{f}^{*}\left(\mathrm{u}_{i} \mathrm{v}_{i}\right)=\mathrm{k}+7 \mathrm{i}-6, \quad 1 \leq \mathrm{i} \leq \mathrm{n}-1$

$\mathrm{f}^{*}\left(\mathrm{u}_{i+1} \mathrm{w}_{i}\right)=\mathrm{k}+7 \mathrm{i}-1,1 \leq \mathrm{i} \leq \mathrm{n}-1$

$\mathrm{f}^{*}\left(\mathrm{v}_{i} \mathrm{w}_{i}\right)=\mathrm{k}+7 \mathrm{i}-4,1 \leq \mathrm{i} \leq \mathrm{n}-1$.

Hence $\mathrm{f}\left[\mathrm{V}\left(\mathrm{Q}_{n}\right)\right] \cup\left\{\mathrm{f}^{*}(\mathrm{e}): \mathrm{e} \in \mathrm{E}\left(\mathrm{Q}_{n}\right)\right\}=\{\mathrm{k}, \mathrm{k}+1, \mathrm{k}+2, \ldots, \mathrm{p}+\mathrm{q}+\mathrm{k}-1\}$.

Therefore any Quadrilateral snake graph $\mathrm{Q}_{n}$ is a k-super cube root cube

mean graph.

An example of 30-Super cube root cube mean labeling of $Q_{5}$ is shown in

Figure 7.

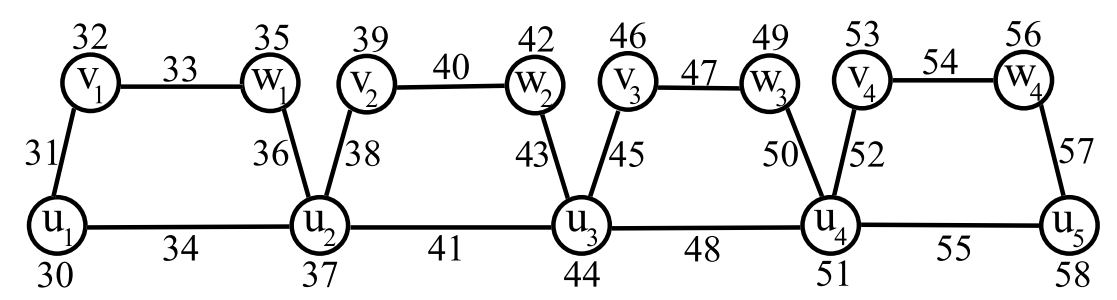

Figure 7: 30-Super cube root cube mean labeling of $Q_{5}$

Theorem 3.6. Any alternate quadrilateral snake graph $A\left(Q_{n}\right)$ is a k-super cube root cube mean graph.

Proof. Let $\mathrm{A}\left(\mathrm{Q}_{n}\right)$ be an alternate quadrilateral snake graph.

In this theorem, consider two cases.

Case 1. Quadrilateral in $A\left(Q_{n}\right)$ starts from $\mathrm{u}_{1}$.

In this case $\mathrm{p}=\left\{\begin{array}{l}2 n, \text { if } n \text { is even; } \\ 2 n-1, \text { if } n \text { is odd. }\end{array}\right.$

$\& \mathrm{q}= \begin{cases}\frac{5 n-2}{2}, & \text { if } n \text { is even } \\ \frac{5 n-5}{2}, & \text { if } n \text { is odd }\end{cases}$ 
Hence $\mathrm{p}+\mathrm{q}= \begin{cases}\frac{9 n-2}{2}, & \text { if } n \text { is even; } \\ \frac{9 n-7}{2}, & \text { if } n \text { is odd. }\end{cases}$

Define a function $\mathrm{f}: \mathrm{V}\left(\mathrm{A}\left(\mathrm{Q}_{n}\right)\right) \rightarrow\{\mathrm{k}, \mathrm{k}+1, \mathrm{k}+2, \ldots, \mathrm{p}+\mathrm{q}+\mathrm{k}-1\}$ by $\mathrm{f}\left(\mathrm{u}_{2 i-1}\right)=\mathrm{k}+9 \mathrm{i}-9,1 \leq \mathrm{i} \leq \frac{n}{2}$ if $\mathrm{n}$ is even $\& 1 \leq \mathrm{i} \leq \frac{n+1}{2}$ if $\mathrm{n}$ is odd. $\mathrm{f}\left(\mathrm{u}_{2 i}\right)=\mathrm{k}+9 \mathrm{i}-2,1 \leq \mathrm{i} \leq \frac{n}{2}$ if $\mathrm{n}$ is even $\& 1 \leq \mathrm{i} \leq \frac{n-1}{2}$ if $\mathrm{n}$ is odd. $\mathrm{f}\left(\mathrm{v}_{i}\right)=\mathrm{k}+9 \mathrm{i}-7,1 \leq \mathrm{i} \leq \frac{n}{2}$ if $\mathrm{n}$ is even $\& 1 \leq \mathrm{i} \leq \frac{n-1}{2}$ if $\mathrm{n}$ is odd. $\mathrm{f}\left(\mathrm{w}_{1}\right)=\left\{\begin{array}{l}k+4, \text { if } k=1,2,3 \\ k+5, \text { otherwise }\end{array}\right.$

$\mathrm{f}\left(\mathrm{w}_{i}\right)=\mathrm{k}+9 \mathrm{i}-4,2 \leq \mathrm{i} \leq \frac{n}{2}$ if $\mathrm{n}$ is even $\& 2 \leq \mathrm{i} \leq \frac{n-1}{2}$ if $\mathrm{n}$ is odd.

Then, the edge labels of $\mathrm{A}\left(\mathrm{Q}_{n}\right)$ are

$\mathrm{f}^{*}\left(\mathrm{u}_{1} \mathrm{u}_{2}\right)=\left\{\begin{array}{l}k+5, \text { if } k=1,2,3 \\ k+4, \text { otherwise. }\end{array}\right.$

$\mathrm{f}^{*}\left(\mathrm{u}_{2 i-1} \mathrm{u}_{2 i}\right)=\mathrm{k}+9 \mathrm{i}-5,2 \leq \mathrm{i} \leq \frac{n}{2}$ if $\mathrm{n}$ is even $\& 2 \leq \mathrm{i} \leq \frac{n-1}{2}$ if $\mathrm{n}$ is odd.

$\mathrm{f}^{*}\left(\mathrm{u}_{2 i} \mathrm{u}_{2 i+1}\right)=\mathrm{k}+9 \mathrm{i}-1,1 \leq \mathrm{i} \leq \frac{n-2}{2}$ if $\mathrm{n}$ is even $\& 1 \leq \mathrm{i} \leq \frac{n-1}{2}$ if $\mathrm{n}$ is odd. $\mathrm{f}^{*}\left(\mathrm{u}_{2 i-1} \mathrm{v}_{i}\right)=\mathrm{k}+9 \mathrm{i}-8,1 \leq \mathrm{i} \leq \frac{n}{2}$ if $\mathrm{n}$ is even $\& 1 \leq \mathrm{i} \leq \frac{n-1}{2}$ if $\mathrm{n}$ is odd. $\mathrm{f}^{*}\left(\mathrm{u}_{2 i} \mathrm{w}_{i}\right)=\mathrm{k}+9 \mathrm{i}-3,1 \leq \mathrm{i} \leq \frac{n}{2}$ if $\mathrm{n}$ is even $\& 1 \leq \mathrm{i} \leq \frac{n-1}{2}$ if $\mathrm{n}$ is odd. $\mathrm{f}^{*}\left(\mathrm{v}_{i} \mathrm{w}_{i}\right)=\mathrm{k}+9 \mathrm{i}-6,1 \leq \mathrm{i} \leq \frac{n}{2}$ if $\mathrm{n}$ is even $\& 1 \leq \mathrm{i} \leq \frac{n-1}{2}$ if $\mathrm{n}$ is odd. Hence $\mathrm{f}\left[\mathrm{V}\left(\mathrm{A}\left(\mathrm{Q}_{n}\right)\right)\right] \cup\left\{\mathrm{f}^{*}(\mathrm{e}): \mathrm{e} \in \mathrm{E}\left(\mathrm{A}\left(\mathrm{Q}_{n}\right)\right)\right\}=\{\mathrm{k}, \mathrm{k}+1, \mathrm{k}+2, \ldots, \mathrm{p}+\mathrm{q}+\mathrm{k}-1\}$. An example of 100-super cube root cube mean labeling of $A\left(\mathrm{Q}_{6}\right)$ [ quadrilateral start from $\mathrm{u}_{1}$ ] is shown in Figure 8.

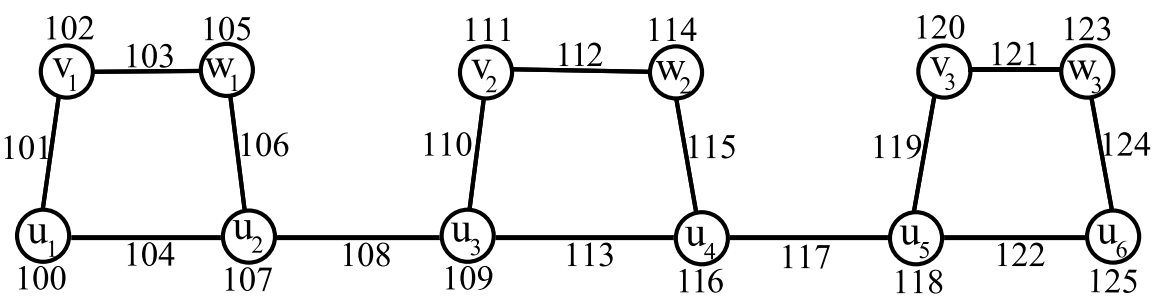

Figure 8: 100-super cube root cube mean labeling of $A\left(Q_{6}\right)$ [quadrilateral start from $\left.u_{1}\right]$ 
Case 2. Quadrilateral in $A\left(Q_{n}\right)$ starts from $u_{2}$

In this case $\mathrm{p}=\left\{\begin{array}{l}2 n-2, \text { if } n \text { is even; } \\ 2 n-1, \text { if } n \text { is odd. }\end{array}\right.$

$\& \mathrm{q}=\left\{\begin{array}{l}\frac{5 n}{2}-4, \text { if } n \text { is even } \\ \frac{5 n-5}{2}, \text { if } n \text { is odd }\end{array}\right.$

Hence $\mathrm{p}+\mathrm{q}=\left\{\begin{array}{l}\frac{9 n-12}{2}, \text { if } n \text { is even } \\ \frac{9 n-7}{2}, \text { if } n \text { is odd. }\end{array}\right.$

Define a function $\mathrm{f}: \mathrm{V}\left(\mathrm{A}\left(\mathrm{Q}_{n}\right)\right) \rightarrow\{\mathrm{k}, \mathrm{k}+1, \mathrm{k}+2, \ldots, \mathrm{p}+\mathrm{q}+\mathrm{k}-1\}$ by

$\mathrm{f}\left(\mathrm{u}_{2 i-1}\right)=\mathrm{k}+9 \mathrm{i}-9,1 \leq \mathrm{i} \leq \frac{n}{2}$ if $\mathrm{n}$ is even $\& 1 \leq \mathrm{i} \leq \frac{n+1}{2}$ if $\mathrm{n}$ is odd.

$\mathrm{f}\left(\mathrm{u}_{2 i}\right)=\mathrm{k}+9 \mathrm{i}-7,1 \leq \mathrm{i} \leq \frac{n}{2}$ if $\mathrm{n}$ is even $\& 1 \leq \mathrm{i} \leq \frac{n-1}{2}$ if $\mathrm{n}$ is odd. $\mathrm{f}\left(\mathrm{v}_{i}\right)$

$=\mathrm{k}+9 \mathrm{i}-5,1 \leq \mathrm{i} \leq \frac{n-2}{2}$ if $\mathrm{n}$ is even $\& 1 \leq \mathrm{i} \leq \frac{n-1}{2}$ if $\mathrm{n}$ is odd.

$\mathrm{f}\left(\mathrm{w}_{1}\right)=\left\{\begin{array}{l}k+6, \text { if } k=1 \\ k+7, \text { otherwise. }\end{array}\right.$

$\mathrm{f}\left(\mathrm{w}_{i}\right)=\mathrm{k}+9 \mathrm{i}-2,2 \leq \mathrm{i} \leq \frac{n-2}{2}$ if $\mathrm{n}$ is even $\& 2 \leq \mathrm{i} \leq \frac{n-1}{2}$ if $\mathrm{n}$ is odd.

Then, the edge labels of $\mathrm{A}\left(\mathrm{Q}_{n}\right)$ are

$\mathrm{f}^{*}\left(\mathrm{u}_{2 i-1} \mathrm{u}_{2 i}\right)=\mathrm{k}+9 \mathrm{i}-8,1 \leq \mathrm{i} \leq \frac{n}{2}$ if $\mathrm{n}$ is even $\& 1 \leq \mathrm{i} \leq \frac{n-1}{2}$ if $\mathrm{n}$ is odd.

$\mathrm{f}^{*}\left(\mathrm{u}_{2} \mathrm{u}_{3}\right)=\left\{\begin{array}{l}k+7, \text { if } k=1 \\ k+6, \text { otherwise }\end{array}\right.$

$\mathrm{f}^{*}\left(\mathrm{u}_{2 i} \mathrm{u}_{2 i+1}\right)=\mathrm{k}+9 \mathrm{i}-3,2 \leq \mathrm{i} \leq \frac{n-2}{2}$ if $\mathrm{n}$ is even $\& 2 \leq \mathrm{i} \leq \frac{n-1}{2}$ if $\mathrm{n}$ is

odd.

$\mathrm{f}^{*}\left(\mathrm{u}_{2 i} \mathrm{v}_{i}\right)=\mathrm{k}+9 \mathrm{i}-6,1 \leq \mathrm{i} \leq \frac{n-2}{2}$ if $\mathrm{n}$ is even $\& 1 \leq \mathrm{i} \leq \frac{n-1}{2}$ if $\mathrm{n}$ is odd.

$\mathrm{f}^{*}\left(\mathrm{u}_{2 i+1} \mathrm{w}_{i}\right)=\mathrm{k}+9 \mathrm{i}-1,1 \leq \mathrm{i} \leq \frac{n-2}{2}$ if $\mathrm{n}$ is even $\& 1 \leq \mathrm{i} \leq \frac{n-1}{2}$ if $\mathrm{n}$ is odd.

$\mathrm{f}^{*}\left(\mathrm{v}_{i} \mathrm{w}_{i}\right)=\mathrm{k}+9 \mathrm{i}-4,1 \leq \mathrm{i} \leq \frac{n-2}{2}$ if $\mathrm{n}$ is even $\& 1 \leq \mathrm{i} \leq \frac{n-1}{2}$ if $\mathrm{n}$ is odd.

Hence $\mathrm{f}\left[\mathrm{V}\left(\mathrm{A}\left(\mathrm{Q}_{n}\right)\right)\right] \cup\left\{\mathrm{f}^{*}(\mathrm{e}): \mathrm{e} \in \mathrm{E}\left(\mathrm{A}\left(\mathrm{Q}_{n}\right)\right)\right\}=\{\mathrm{k}, \mathrm{k}+1, \mathrm{k}+2, \ldots, \mathrm{p}+\mathrm{q}+\mathrm{k}-1\}$. 
An example of 100-super cube root cube mean labeling of $A\left(Q_{7}\right)$ [ quadrilateral start from $\mathrm{u}_{2}$ ] is shown in Figure 9.

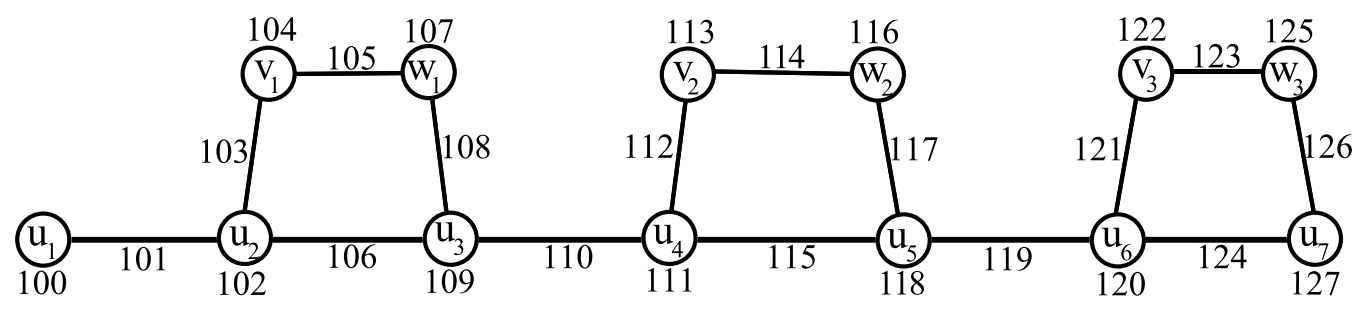

Figure 9: 100-super cube root cube mean labeling of $A\left(Q_{7}\right)$ [quadrilateral start from $u_{2}$ ]

From the above cases, an alternate quadrilateral snake graph $A\left(Q_{n}\right)$ is a k-super cube root cube mean graph.

Theorem 3.7. Any double quadrilateral snake graph $D\left(Q_{n}\right)$ is a $k$-super cube root cube mean graph.

Proof. Let $\mathrm{D}\left(\mathrm{Q}_{n}\right)$ be a double quadrilateral snake graph.

Here $\mathrm{p}=5 \mathrm{n}-4 \& \mathrm{q}=7 \mathrm{n}-7$

Hence $\mathrm{p}+\mathrm{q}=12 \mathrm{n}-11$.

Define a function $\mathrm{f}: \mathrm{V}\left(\mathrm{D}\left(\mathrm{Q}_{n}\right)\right) \rightarrow\{\mathrm{k}, \mathrm{k}+1, \mathrm{k}+2, \ldots, \mathrm{p}+\mathrm{q}+\mathrm{k}-1\}$ by $\mathrm{f}\left(\mathrm{u}_{i}\right)=\mathrm{k}+12 \mathrm{i}-12,1 \leq \mathrm{i} \leq \mathrm{n}$

$\mathrm{f}\left(\mathrm{v}_{i}\right)=\mathrm{k}+12 \mathrm{i}-10,1 \leq \mathrm{i} \leq \mathrm{n}-1$

$\mathrm{f}\left(\mathrm{w}_{i}\right)=\mathrm{k}+12 \mathrm{i}-7,1 \leq \mathrm{i} \leq \mathrm{n}-1$

$\mathrm{f}\left(v_{i}^{\prime}\right)=\mathrm{k}+12 \mathrm{i}-6,1 \leq \mathrm{i} \leq \mathrm{n}-1$

$\mathrm{f}\left(w_{1}^{\prime}\right)=\left\{\begin{array}{l}k+8, \text { if } k=1,2,3, \ldots, 9 ; \\ k+10, \text { otherwise. }\end{array}\right.$

$\mathrm{f}\left(w_{i}^{\prime}\right)=\mathrm{k}+12 \mathrm{i}-2,2 \leq \mathrm{i} \leq \mathrm{n}-1$.

Then, the edge labels of $\mathrm{D}\left(\mathrm{Q}_{n}\right)$ are

$\mathrm{f}^{*}\left(\mathrm{u}_{1} \mathrm{u}_{2}\right)=\left\{\begin{array}{l}k+9, \text { if } k=1,2,3, \ldots, 9 \\ k+7, \text { otherwise }\end{array}\right.$ 
$\mathrm{f}^{*}\left(\mathrm{u}_{i} \mathrm{u}_{i+1}\right)=\mathrm{k}+12 \mathrm{i}-5,2 \leq \mathrm{i} \leq \mathrm{n}-1$

$\mathrm{f}^{*}\left(\mathrm{u}_{i} \mathrm{v}_{i}\right)=\mathrm{k}+12 \mathrm{i}-11,1 \leq \mathrm{i} \leq \mathrm{n}-1$

$\mathrm{f}^{*}\left(\mathrm{u}_{2} \mathrm{w}_{1}\right)=\left\{\begin{array}{l}k+10, \text { if } k=1,2,3, \ldots, 9 ; \\ k+9, \text { otherwise }\end{array}\right.$

$\mathrm{f}^{*}\left(\mathrm{u}_{i+1} \mathrm{w}_{i}\right)=\mathrm{k}+12 \mathrm{i}-3,2 \leq \mathrm{i} \leq \mathrm{n}-1$

$\mathrm{f}^{*}\left(\mathrm{v}_{i} \mathrm{w}_{i}\right)=\mathrm{k}+12 \mathrm{i}-9, \quad 1 \leq \mathrm{i} \leq \mathrm{n}-1$

$\mathrm{f}^{*}\left(\mathrm{u}_{i} \mathrm{v}_{i}^{\prime}\right)=\mathrm{k}+12 \mathrm{i}-8, \quad 1 \leq \mathrm{i} \leq \mathrm{n}-1$

$\mathrm{f}^{*}\left(\mathrm{u}_{i+1} \mathrm{w}_{i}^{\prime}\right)=\mathrm{k}+12 \mathrm{i}-1, \quad 1 \leq \mathrm{i} \leq \mathrm{n}-1$

$\mathrm{f}^{*}\left(\mathrm{v}_{1}^{\prime} \mathrm{w}_{1}^{\prime}\right)=\left\{\begin{array}{l}k+7, \text { if } k=1,2,3, \ldots, 9 ; \\ k+8, \text { otherwise. }\end{array}\right.$

$\mathrm{f}^{*}\left(\mathrm{v}_{i}^{\prime} \mathrm{w}_{i}^{\prime}\right)=\mathrm{k}+12 \mathrm{i}-4,2 \leq \mathrm{i} \leq \mathrm{n}-1$

Hence $\mathrm{f}\left[\mathrm{V}\left(\mathrm{D}\left(\mathrm{Q}_{n}\right)\right)\right] \cup\left\{\mathrm{f}^{*}(\mathrm{e}): \mathrm{e} \in \mathrm{E}\left(\mathrm{D}\left(\mathrm{Q}_{n}\right)\right)\right\}=\{\mathrm{k}, \mathrm{k}+1, \mathrm{k}+2, \ldots, \mathrm{p}+\mathrm{q}+\mathrm{k}-1\}$.

Therefore any double quadrilateral snake graph $\mathrm{D}\left(\mathrm{Q}_{n}\right)$ is a $\mathrm{k}$-super cube root cube mean graph.

An example of 25- Super cube root cube mean labeling of $\mathrm{D}\left(\mathrm{Q}_{5}\right)$ is shown

in Figure 10.

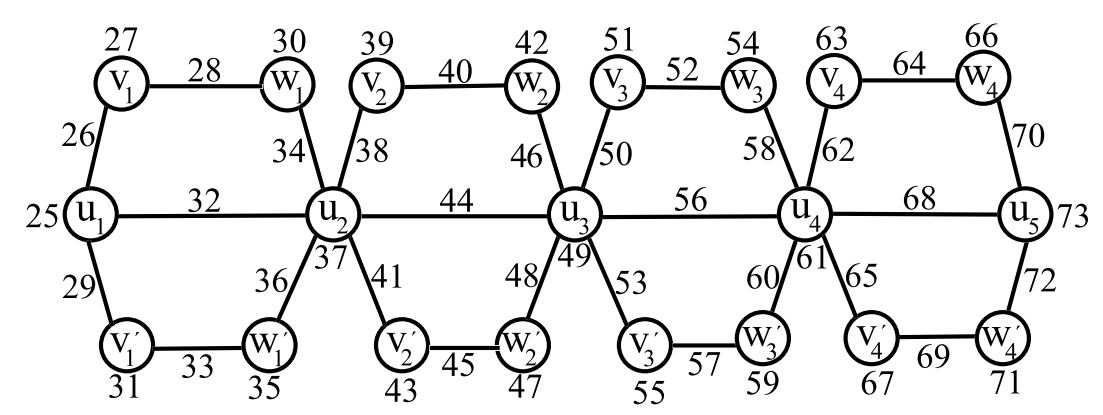

Figure 10: caption25- Super cube root cube mean labeling of $D\left(Q_{5}\right)$

Theorem 3.8. Any alternate double quadrilateral snake graph $A D\left(Q_{n}\right)$ is a k-super cube root cube mean graph. 
Proof. Let $\mathrm{AD}\left(\mathrm{Q}_{n}\right)$ be an alternate double quadrilateral snake graph In this theorem, consider two cases.

Case 1. Quadrilateral in $\operatorname{AD}\left(Q_{n}\right)$ starts from $u_{1}$

In this case $\mathrm{p}=\left\{\begin{array}{l}3 n, \text { if } n \text { is even; } \\ 3 n-2, \text { if } n \text { is odd. }\end{array}\right.$

$\& \mathrm{q}=\left\{\begin{array}{l}4 n-1, \text { if } n \text { is even } \\ 4 n-4, \text { if } n \text { is odd }\end{array}\right.$

Hence $\mathrm{p}+\mathrm{q}=\left\{\begin{array}{l}7 n-1, \text { if } n \text { is even; } \\ 7 n-6, \text { if } n \text { is odd. }\end{array}\right.$

Define a function $\mathrm{f}: \mathrm{V}\left(\mathrm{AD}\left(\mathrm{Q}_{n}\right)\right) \rightarrow\{\mathrm{k}, \mathrm{k}+1, \mathrm{k}+2, \ldots, \mathrm{p}+\mathrm{q}+\mathrm{k}-1\}$

by

$\mathrm{f}\left(\mathrm{u}_{2 i-1}\right)=\mathrm{k}+14 \mathrm{i}-14,1 \leq \mathrm{i} \leq \frac{n}{2}$ if $\mathrm{n}$ is even $\& 1 \leq \mathrm{i} \leq \frac{n+1}{2}$ if $\mathrm{n}$ is odd. $\mathrm{f}\left(\mathrm{u}_{2 i}\right)=\mathrm{k}+14 \mathrm{i}-2,1 \leq \mathrm{i} \leq \frac{n}{2}$ if $\mathrm{n}$ is even $\& 1 \leq \mathrm{i} \leq \frac{n-1}{2}$ if $\mathrm{n}$ is odd.

$\mathrm{f}\left(\mathrm{v}_{i}\right)=\mathrm{k}+14 \mathrm{i}-12,1 \leq \mathrm{i} \leq \frac{n}{2}$ if $\mathrm{n}$ is even $\& 1 \leq \mathrm{i} \leq \frac{n-1}{2}$ if $\mathrm{n}$ is odd.

$\mathrm{f}\left(\mathrm{w}_{i}\right)=\mathrm{k}+14 \mathrm{i}-9,1 \leq \mathrm{i} \leq \frac{n}{2}$ if $\mathrm{n}$ is even $\& 1 \leq \mathrm{i} \leq \frac{n-1}{2}$ if $\mathrm{n}$ is odd.

$\mathrm{f}\left(\mathrm{v}_{i}^{\prime}\right)=\mathrm{k}+14 \mathrm{i}-8,1 \leq \mathrm{i} \leq \frac{n}{2}$ if $\mathrm{n}$ is even $\& 1 \leq \mathrm{i} \leq \frac{n-1}{2}$ if $\mathrm{n}$ is odd.

$\mathrm{f}\left(\mathrm{w}_{1}^{\prime}\right)=\left\{\begin{array}{l}k+8, \text { if } k=1,2,3, \ldots, 9 \\ k+10, \text { otherwise }\end{array}\right.$

$\mathrm{f}\left(\mathrm{w}_{i}^{\prime}\right)=\mathrm{k}+14 \mathrm{i}-4,2 \leq \mathrm{i} \leq \frac{n}{2}$ if $\mathrm{n}$ is even $\& 2 \leq \mathrm{i} \leq \frac{n-1}{2}$ if $\mathrm{n}$ is odd.

Then, the edge labels of $\mathrm{AD}\left(\mathrm{Q}_{n}\right)$ are

$\mathrm{f}^{*}\left(\mathrm{u}_{1} \mathrm{u}_{2}\right)=\left\{\begin{array}{l}k+9, \text { if } k=1,2, \ldots, 9 \\ k+7, \text { otherwise. }\end{array}\right.$

$\mathrm{f}^{*}\left(\mathrm{u}_{2 i-1} \mathrm{u}_{2 i}\right)=\mathrm{k}+14 \mathrm{i}-7,2 \leq \mathrm{i} \leq \frac{n}{2}$ if $\mathrm{n}$ is even $\& 2 \leq \mathrm{i} \leq \frac{n-1}{2}$ if $\mathrm{n}$ is odd.

$\mathrm{f}^{*}\left(\mathrm{u}_{2 i} \mathrm{u}_{2 i+1}\right)=\mathrm{k}+14 \mathrm{i}-1,1 \leq \mathrm{i} \leq \frac{n-2}{2}$ if $\mathrm{n}$ is even $\& 1 \leq \mathrm{i} \leq \frac{n-1}{2}$ if $\mathrm{n}$ is odd.

$\mathrm{f}^{*}\left(\mathrm{u}_{2 i-1} \mathrm{v}_{i}\right)=\mathrm{k}+14 \mathrm{i}-13,1 \leq \mathrm{i} \leq \frac{n}{2}$ if $\mathrm{n}$ is even $\& 1 \leq \mathrm{i} \leq \frac{n-1}{2}$ if $\mathrm{n}$ is odd. $\mathrm{f}^{*}\left(\mathrm{u}_{2} \mathrm{w}_{1}\right)=\left\{\begin{array}{l}k+10, \text { if } k=1,2, \ldots, 9 ; \\ k+9, \text { otherwise. }\end{array}\right.$ 
$\mathrm{f}^{*}\left(\mathrm{u}_{2 i} \mathrm{w}_{i}\right)=\mathrm{k}+14 \mathrm{i}-5,2 \leq \mathrm{i} \leq \frac{n}{2}$ if $\mathrm{n}$ is even $\& 2 \leq \mathrm{i} \leq \frac{n-1}{2}$ if $\mathrm{n}$ is odd.

$\mathrm{f}^{*}\left(\mathrm{v}_{i} \mathrm{w}_{i}\right)=\mathrm{k}+14 \mathrm{i}-11,1 \leq \mathrm{i} \leq \frac{n}{2}$ if $\mathrm{n}$ is even $\& 1 \leq \mathrm{i} \leq \frac{n-1}{2}$ if $\mathrm{n}$ is odd.

$\mathrm{f}^{*}\left(\mathrm{u}_{2 i-1} \mathrm{v}_{i}^{\prime}\right)=\mathrm{k}+14 \mathrm{i}-10,1 \leq \mathrm{i} \leq \frac{n}{2}$ if $\mathrm{n}$ is even $\& 1 \leq \mathrm{i} \leq \frac{n-1}{2}$ if $\mathrm{n}$ is odd.

$\mathrm{f}^{*}\left(\mathrm{u}_{2 i} \mathrm{w}_{i}^{\prime}\right)=\mathrm{k}+14 \mathrm{i}-3,1 \leq \mathrm{i} \leq \frac{n}{2}$ if $\mathrm{n}$ is even $\& 1 \leq \mathrm{i} \leq \frac{n-1}{2}$ if $\mathrm{n}$ is odd.

$\mathrm{f}^{*}\left(\mathrm{v}_{1}^{\prime} \mathrm{w}_{1}^{\prime}\right)=\left\{\begin{array}{l}k+7, \text { if } k=1,2, \ldots, 9 ; \\ k+8, \text { otherwise. }\end{array}\right.$

$\mathrm{f}^{*}\left(\mathrm{v}_{i}^{\prime} \mathrm{w}_{i}^{\prime}\right)=\mathrm{k}+14 \mathrm{i}-6,2 \leq \mathrm{i} \leq \frac{n}{2}$ if $\mathrm{n}$ is even $\& 2 \leq \mathrm{i} \leq \frac{n-1}{2}$ if $\mathrm{n}$ is odd.

Hence $\mathrm{f}\left[\mathrm{V}\left(\mathrm{AD}\left(\mathrm{Q}_{n}\right)\right)\right] \cup\left\{\mathrm{f}^{*}(\mathrm{e}): \mathrm{e} \in \mathrm{E}\left(\mathrm{AD}\left(\mathrm{Q}_{n}\right)\right)\right\}=\{\mathrm{k}, \mathrm{k}+1, \mathrm{k}+2, \ldots, \mathrm{p}+\mathrm{q}+\mathrm{k}-1\}$.

An example of 115-super cube root cube mean labeling of $A D\left(Q_{6}\right)$ [quadrilateral start from $u_{1}$ ] is shown in Figure 11.

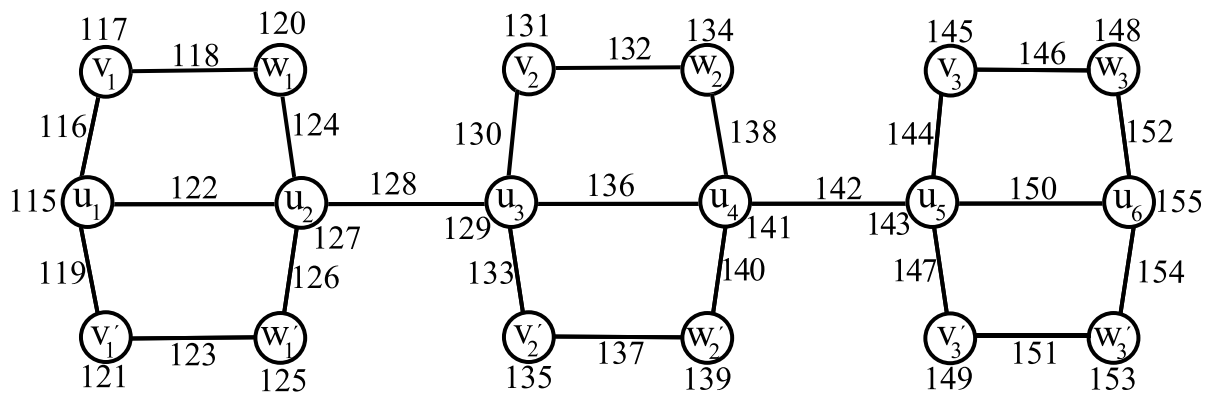

Figure 11: 115-super cube root cube mean labeling of $A D\left(Q_{6}\right)$ [quadrilateral start from $u_{1}$ ]

Case 2. Quadrilateral in $\operatorname{AD}\left(Q_{n}\right)$ starts from $u_{2}$

In this case $\mathrm{p}=\left\{\begin{array}{l}3 n-4, \text { if } n \text { is even; } \\ 3 n-2, \text { if } n \text { is odd. }\end{array}\right.$

$\& \mathrm{q}=\left\{\begin{array}{l}4 n-7, \text { if } n \text { is even } \\ 4 n-4, \text { if } n \text { is odd }\end{array}\right.$

Hence $\mathrm{p}+\mathrm{q}=\left\{\begin{array}{l}7 n-11, \text { if } n \text { is even } \\ 7 n-6, \text { if } n \text { is odd. }\end{array}\right.$ 
Define a function $\mathrm{f}: \mathrm{V}\left(\mathrm{AD}\left(\mathrm{Q}_{n}\right)\right) \rightarrow\{\mathrm{k}, \mathrm{k}+1, \mathrm{k}+2, \ldots, \mathrm{p}+\mathrm{q}+\mathrm{k}-1\}$ by

$\mathrm{f}\left(\mathrm{u}_{2 i-1}\right)=\mathrm{k}+14 \mathrm{i}-14,1 \leq \mathrm{i} \leq \frac{n}{2}$ if $\mathrm{n}$ is even $\& 1 \leq \mathrm{i} \leq \frac{n+1}{2}$ if $\mathrm{n}$ is odd. $\mathrm{f}\left(\mathrm{u}_{2 i}\right)=\mathrm{k}+14 \mathrm{i}-12,1 \leq \mathrm{i} \leq \frac{n}{2}$ if $\mathrm{n}$ is even $\& 1 \leq \mathrm{i} \leq \frac{n-1}{2}$ if $\mathrm{n}$ is odd. $\mathrm{f}\left(\mathrm{v}_{i}\right)=\mathrm{k}+14 \mathrm{i}-10,1 \leq \mathrm{i} \leq \frac{n-2}{2}$ if $\mathrm{n}$ is even $\& 1 \leq \mathrm{i} \leq \frac{n-1}{2}$ if $\mathrm{n}$ is odd. $\mathrm{f}\left(\mathrm{w}_{i}\right)=\mathrm{k}+14 \mathrm{i}-7,1 \leq \mathrm{i} \leq \frac{n-2}{2}$ if $\mathrm{n}$ is even $\& 1 \leq \mathrm{i} \leq \frac{n-1}{2}$ if $\mathrm{n}$ is odd. $\mathrm{f}\left(\mathrm{v}_{i}^{\prime}\right)=\mathrm{k}+14 \mathrm{i}-6,1 \leq \mathrm{i} \leq \frac{n-2}{2}$ if $\mathrm{n}$ is even $\& 1 \leq \mathrm{i} \leq \frac{n-1}{2}$ if $\mathrm{n}$ is odd. $\mathrm{f}\left(\mathrm{w}_{1}^{\prime}\right)=\left\{\begin{array}{l}k+11, \text { if } k=1,2,3, \ldots, 7 \\ k+12, \text { otherwise }\end{array}\right.$

$\mathrm{f}\left(\mathrm{w}_{i}^{\prime}\right)=\mathrm{k}+14 \mathrm{i}-2, \quad 2 \leq \mathrm{i} \leq \frac{n-2}{2}$ if $\mathrm{n}$ is even $\& 2 \leq \mathrm{i} \leq \frac{n-1}{2}$ if $\mathrm{n}$ is odd.

Then, the edge labels of $\mathrm{AD}\left(\mathrm{Q}_{n}\right)$ are $\mathrm{f}^{*}\left(\mathrm{u}_{2 i-1} \mathrm{u}_{2 i}\right)=\mathrm{k}+14 \mathrm{i}-13,1 \leq \mathrm{i} \leq \frac{n}{2}$ if $\mathrm{n}$ is even $\& 1 \leq \mathrm{i} \leq \frac{n-1}{2}$ if $\mathrm{n}$ is odd. $\mathrm{f}^{*}\left(\mathrm{u}_{2} \mathrm{u}_{3}\right)=\left\{\begin{array}{l}k+10, \text { if } k=1,2, \ldots, 7 \\ k+9, \text { otherwise }\end{array}\right.$

$\mathrm{f}^{*}\left(\mathrm{u}_{2 i} \mathrm{u}_{2 i+1}\right)=\mathrm{k}+14 \mathrm{i}-5,2 \leq \mathrm{i} \leq \frac{n-2}{2}$ if $\mathrm{n}$ is even $\& 2 \leq \mathrm{i} \leq \frac{n-1}{2}$ if $\mathrm{n}$ is odd.

$\mathrm{f}^{*}\left(\mathrm{u}_{2 i} \mathrm{v}_{i}\right)=\mathrm{k}+14 \mathrm{i}-11, \quad 1 \leq \mathrm{i} \leq \frac{n-2}{2}$ if $\mathrm{n}$ is even $\& 1 \leq \mathrm{i} \leq \frac{n-1}{2}$ if $\mathrm{n}$ is odd. $\mathrm{f}^{*}\left(\mathrm{u}_{3} \mathrm{w}_{1}\right)=\left\{\begin{array}{l}k+12, \text { if } k=1,2, \ldots, 7 \\ k+11, \text { otherwise }\end{array}\right.$

$\mathrm{f}^{*}\left(\mathrm{u}_{2 i+1} \mathrm{w}_{i}\right)=\mathrm{k}+14 \mathrm{i}-3,2 \leq \mathrm{i} \leq \frac{n-2}{2}$ if $\mathrm{n}$ is even $\& 2 \leq \mathrm{i} \leq \frac{n-1}{2}$ if $\mathrm{n}$ is odd.

$\mathrm{f}^{*}\left(\mathrm{v}_{i} \mathrm{w}_{i}\right)=\mathrm{k}+14 \mathrm{i}-9,1 \leq \mathrm{i} \leq \frac{n-2}{2}$ if $\mathrm{n}$ is even $\& 1 \leq \mathrm{i} \leq \frac{n-1}{2}$ if $\mathrm{n}$ is odd. $\mathrm{f}^{*}\left(\mathrm{u}_{2 i} \mathrm{v}_{i}^{\prime}\right)=\mathrm{k}+14 \mathrm{i}-8,1 \leq \mathrm{i} \leq \frac{n-2}{2}$ if $\mathrm{n}$ is even $\& 1 \leq \mathrm{i} \leq \frac{n-1}{2}$ if $\mathrm{n}$ is odd. $\mathrm{f}^{*}\left(\mathrm{u}_{2 i+1} \mathrm{w}_{i}^{\prime}\right)=\mathrm{k}+14 \mathrm{i}-1, \quad 1 \leq \mathrm{i} \leq \frac{n-2}{2}$ if $\mathrm{n}$ is even $\& 1 \leq \mathrm{i} \leq \frac{n-1}{2}$ if $\mathrm{n}$ is odd.

$\mathrm{f}^{*}\left(\mathrm{v}_{1}^{\prime} \mathrm{w}_{1}^{\prime}\right)=\left\{\begin{array}{l}k+9, \text { if } k=1,2, \ldots, 7 \\ k+10, \text { otherwise. }\end{array}\right.$

$\mathrm{f}^{*}\left(\mathrm{v}_{i}^{\prime} \mathrm{w}_{i}^{\prime}\right)=\mathrm{k}+14 \mathrm{i}-4,2 \leq \mathrm{i} \leq \frac{n-2}{2}$ if $\mathrm{n}$ is even $\& 2 \leq \mathrm{i} \leq \frac{n-1}{2}$ if $\mathrm{n}$ is odd.

Hence $\mathrm{f}\left[\mathrm{V}\left(\mathrm{AD}\left(\mathrm{Q}_{n}\right)\right)\right] \cup\left\{\mathrm{f}^{*}(\mathrm{e}): \mathrm{e} \in \mathrm{E}\left(\mathrm{AD}\left(\mathrm{Q}_{n}\right)\right)\right\}=\{\mathrm{k}, \mathrm{k}+1, \mathrm{k}+2, \ldots, \mathrm{p}+\mathrm{q}+\mathrm{k}-1\}$. An example of 115 -super cube root cube mean labeling of $\mathrm{AD}\left(\mathrm{Q}_{7}\right)$ [ quadri- 
lateral start from $\mathrm{u}_{2}$ ] is shown in Figure 12.

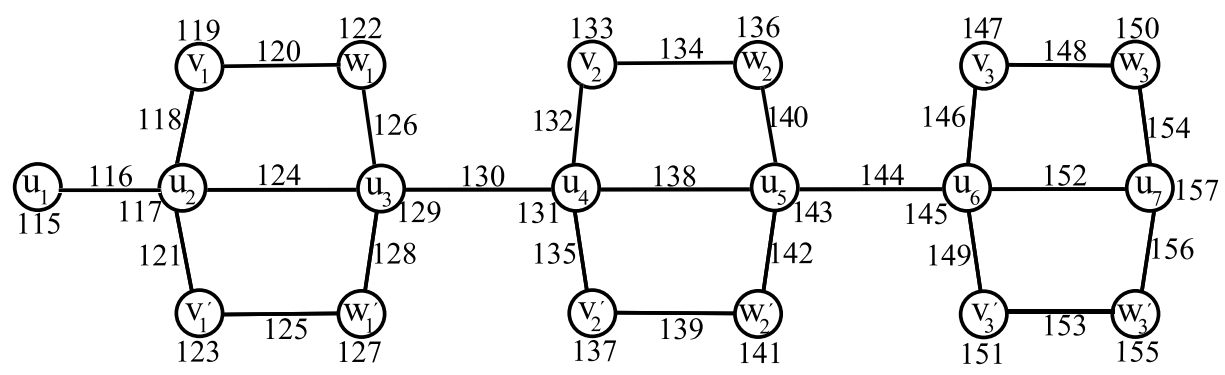

Figure 12: 115- super cube root cube mean labeling of $A D\left(Q_{7}\right)$ [quadrilateral start from $u_{2}$ ]

From the above cases, an alternate double quadrilateral snake graph $\mathrm{AD}\left(\mathrm{Q}_{n}\right)$ is a k-super cube root cube mean graph.

\section{References}

[1] K. Akilandeswari, "Super Root Square Mean Labeling of Some Graphs", International Journal of Science and Research (IJSR), vol. 6, no. 2, 2017. [On line]. Available: https:// bit.ly/ 2YxOkZB

[2] J. A. Gallian, "A dynamic survey of graph labeling", The Electronic Journal of Combinatorics, \# DS6., 2019, [On line]. Available: https:/ / bit.ly/ 2AojJ92

[3] P. Jeyanthi, D. Ramya and P. Thangavelu, "On super mean labeling of some graphs", SUT Journal of Mathematics, vol. 46, no. 1, pp. 53-66, 2010. [On line]. Available: https:/ / bit.ly/ 2UHqOrX

[4] S. Kulandhai Therese and K. Romila, "Cube root Cube Mean labeling of Graphs", International Journal of Mathematics Trends and Technology (IJMTT), vol. 65, no. 2, 2019. [On line]. Available: https:/ / bit.ly/ 30Gy1wq 
[5] V. S. Radhika and A. Vijayan, "Super Cube root Cube Mean labeling of Graphs", Journal of Science and Technology, vol. 5, pp. 17-24, 2020. [On line]. Available: https:/ / bit.ly/ 30LAghI

[6] S. Sandhya, S. Somasundaram and S. Anusa, "Some More Results on Root Square mean Graphs", Journal of Mathematics Research, vol. 7, no. 1, pp. 72-81, 2015. [On line]. Available: https:/ / bit.ly/ 30DTCpd

[7] M. Tamilselvi, K. Akilandeswari, and V. Suguna, "k-Super Mean Labeling of Some Graphs", International journal of Science and Research (IJSR), vol. 5, no. 6, 2016. [On line]. Available: https:/ / bit.ly/ 2UEeMzu

\author{
V. Princy Kala \\ Department of Mathematics, \\ Holy Cross College (Autonomous), \\ Nagercoil-629 004, TN, \\ India \\ e-mail: princykala@holycrossngl.edu.in \\ orcid.org/0000-0001-6264-1100
}

